\title{
Comparison of depressive episodes in bipolar disorder and in major depressive disorder within bipolar disorder pedigrees ${ }^{\dagger}$
}

Philip B. Mitchell, Andrew Frankland, Dusan Hadzi-Pavlovic, Gloria Roberts, Justine Corry, Adam Wright, Colleen K. Loo and Michael Breakspear

\section{Background}

Although genetic epidemiological studies have confirmed increased rates of major depressive disorder among the relatives of people with bipolar affective disorder, no report has compared the clinical characteristics of depression between these two groups.

\section{Aims}

To compare clinical features of depressive episodes across participants with major depressive disorder and bipolar disorder from within bipolar disorder pedigrees, and assess the utility of a recently proposed probabilistic approach to distinguishing bipola from unipolar depression. A secondary aim was to identify subgroups within the relatives with major depression potentially indicative of 'genetic' and 'sporadic' subgroups.

\section{Method}

Patients with bipolar disorder types 1 and $2(n=246)$ and patients with major depressive disorder from bipolar pedigrees $(n=120)$ were assessed using the Diagnostic Interview for Genetic Studies. Logistic regression was used to identify distinguishing clinical features and assess the utility of the probabilistic approach. Hierarchical cluster analysis was used to identify subgroups within the major depressive disorder sample.

\section{Results}

Bipolar depression was characterised by significantly higher rates of psychomotor retardation, difficulty thinking, early morning awakening, morning worsening and psychotic features. Depending on the threshold employed, the probabilistic approach yielded a positive predictive value ranging from $74 \%$ to $82 \%$. Two clusters within the major depressive disorder sample were found, one of which demonstrated features characteristic of bipolar depression, suggesting a possible 'genetic' subgroup.

\section{Conclusions}

A number of previously identified clinical differences between unipolar and bipolar depression were confirmed among participants from within bipolar disorder pedigrees, Preliminary validation of the probabilistic approach in differentiating between unipolar and bipolar depression is consistent with dimensional distinctions between the two disorders and offers clinical utility in identifying patients who may warrant further assessment for bipolarity. The major depressive disorder clusters potentially reflect genetic and sporadic subgroups which, if replicated independently, might enable an improved phenotypic definition of underlying bipolarity in genetic analyses.

\section{Declaration of interest}

C.K.L. has received lecture honoraria from Eli Lilly and Wyeth, and grant support from Neuronetics.
There is growing interest in the recognition and treatment of bipolar depression. ${ }^{1,2}$ Although genetic epidemiological studies have confirmed increased rates of major depressive disorder among the relatives of people with bipolar affective disorder, ${ }^{3-5}$ no report has compared the clinical characteristics of depression between these individuals and their relatives with bipolar disorder within the same pedigrees. Confirming differences in these subgroups could be highly informative, first in the debate over phenomenological differences between bipolar disorder and major depression, ${ }^{6-8}$ and second regarding which cases of major depressive disorder in bipolar disorder pedigrees have more 'genetic' or 'sporadic' aetiologies. ${ }^{3,9}$ Given the overlapping presentations of the two forms of depression, the predominance of depressive features among people with bipolar disorder ${ }^{10}$ and the implications of misdiagnosis, ${ }^{11,12}$ the usefulness of a method for distinguishing between the two conditions based on clinically observable depressive features is clear. Recently published guidelines from the International Society for Bipolar Disorders (ISBD) Diagnostic Task Force have argued for a dimensional rather than categorical distinction between bipolar depression and major depressive disorder, ${ }^{8,13}$ leading to the development of a 'probabilistic' approach to the diagnosis of bipolar depression. ${ }^{8}$

See editorial, pp. 272-274, this issue.
A range of depressive features and symptoms more likely to be associated with a diagnosis of bipolar disorder type 1 were identified from the literature in the development of those guidelines (see Appendix), with the presence of five or more specific features being proposed as indicative of bipolar depression, although this cut-off has yet to be empirically validated. In a similar vein, focusing on the finding of a greater prevalence of major depressive disorder among relatives of bipolar disorder probands and the implications of this for linkage studies, Blacker et al estimated that $65-74 \%$ of these relatives with major depressive disorder had genetically determined illness. ${ }^{3,14}$ They proposed developing an index of 'genetic bipolarity' for these individuals, based on a combination of potentially discriminating phenomenological and longitudinal characteristics (not dissimilar to that proposed in the ISBD guidelines). To assess the nature of depressive episodes in both bipolar disorder and major depressive disorder within bipolar disorder pedigrees, we examined a data-set of participants in a molecular genetics study of bipolar disorder. We also tested the utility of the ISBD probabilistic approach in identifying bipolar depression in this sample, ${ }^{8}$ and sought to identify subgroups within the major depressive disorder cases which could represent sporadic $v$. genetically driven forms of illness, i.e. a potential phenotype for underlying bipolarity. 


\section{Method}

Participants were recruited through the Australian Bipolar Disorder Molecular Genetics Study, ${ }^{15}$ undertaken at the University of New South Wales in collaboration with the Prince of Wales Medical Research Institute, the Garvan Institute of Medical Research and Macquarie University in Sydney, Australia. A total of 1128 individuals were recruited in medium-to-large unilineal multigenerational bipolar pedigrees. Initial data on family and illness history were obtained from the proband using the Family Interview for Genetic Studies, ${ }^{16}$ and each participating family member was subsequently assessed using the Diagnostic Interview for Genetic Studies (DIGS) version 2.0. ${ }^{17}$ Interviews were conducted by experienced medical practitioners, psychologists and psychiatric nurses who received training in the use of each instrument. Best-estimate Research Diagnostic Criteria and DSM-IV diagnoses were made by senior research psychiatrists after independent evaluation of DIGS interviews, family informant data and medical records. ${ }^{18}$ The study was approved by the human research ethics committee of the University of New South Wales, and complies with the guidelines of the Australian National Health and Medical Research Council. The sample used did not overlap with the bipolar disorder and major depressive disorder samples described in previous studies by our group, which investigated phenomenological differences between the two forms of depression. ${ }^{19,20}$

\section{Inclusion criteria}

Inclusion in the analysis was restricted to participants with a bestestimate DSM-IV diagnosis of bipolar type 1 disorder, bipolar type 2 disorder or major depressive disorder, the last diagnosis requiring a history of at least two major depressive episodes. For the original molecular genetics study, written informed consent was obtained after complete description to the participants.

\section{Statistical analysis}

For this study we combined participants with bipolar disorder types 1 and 2 into a single category as there were relatively few with type 2 disorder. Continuous variables were non-normally distributed, and comparisons were made using the MannWhitney $U$-test. Categorical data were compared using Pearson's $\chi^{2}$ test. All symptoms significantly associated with diagnosis at the bivariate level were included in binary logistic regression models, to identify whether weighted combinations of symptoms could differentiate bipolar disorder from major depression. Each symptom was entered as a single predictor, and then in a multivariate model to assess independent associations with diagnosis after accounting for the effects of other symptoms. Results are reported as age- and gender-adjusted odds ratios for a diagnosis of bipolar disorder. All analyses were carried out using Stata version 10 on Windows XP.

To assess the utility of the probabilistic approach in identifying bipolar depression, we included nine clinical features and symptoms from the current data-set that had previously been included in the operational criteria for the probabilistic approach (see Appendix). ${ }^{8}$ The DIGS featured items that related to hypersomnia, hyperphagia, weight gain, psychomotor retardation, delusions and hallucinations, pathological guilt, mixed features, early onset and multiple episodes. As all participants had a family history of bipolar disorder, we were unable to include this as a potential distinguishing feature. The number of positive 'bipolar' features was summed for each participant, ranging from 0 to 9 . For each possible score we compared sensitivity and specificity against a diagnostic standard (best-estimate diagnosis), and plotted receiver operating characteristics curves.

To identify subgroups among the major depressive disorder cases that might correspond to either 'genetic' or 'sporadic' depression, propensity scores were calculated for each participant, which were the estimated probabilities of bipolar $v$. unipolar group membership from the previously described bivariate logistic regression. The propensity score, ranging from 0 to 1 , is an index of the probability of 'caseness', or a diagnosis of bipolar disorder, with a higher score indicating a greater likelihood that the individual has the disorder. We predicted that major depressive disorder cases with an underlying bipolar genetic liability would more closely resemble the 'true' bipolar disorder cases than the major depressive disorder cases with a 'sporadic' depression, and therefore have higher propensity scores. Consistent with the probabilistic approach, these cases would be more likely to show clinical and phenomenological features typically associated with bipolar depression, based on the stronger underlying genetic component to their illness. After calculating propensity scores, we carried out a hierarchical cluster analysis, using betweengroups linkage, to identify any groupings within the major depressive disorder cases, and compared clinical feature and symptom profiles between clusters.

\section{Results}

Of the 1128 participants, $423(37.5 \%)$ met initial diagnostic criteria. Of these, 57 had never had a major depressive episode (i.e. they reported only manic episodes) or provided incomplete data on depressive episodes for the DIGS interview. Of the final 366 participants included in the analysis, $202(55.2 \%)$ had been diagnosed with DSM-IV bipolar type 1 disorder, 44 (12.0\%) with bipolar type 2 disorder and 120 (32.7\%) with major depressive disorder. All participants with major depressive disorder had at least one first-degree relative with type 1 bipolar disorder.

\section{Sociodemographic characteristics}

The sociodemographic characteristics of the sample are reported in Table 1; no significant difference was observed between the two diagnostic groups in age or gender ratio.

\section{Longitudinal characteristics}

Data on the illness course and clinical characteristics of bipolar disorder and major depressive disorder participants are shown in Table 1. Bipolar disorder was significantly associated with a greater number of lifetime depressive episodes, with a higher proportion of participants with bipolar disorder (48.4\%) reporting at least five lifetime depressive episodes compared with the major depressive disorder group (26.4\%). No difference was found in either age at onset or duration of the most severe depressive episode.

\section{Treatment and suicidal behaviour}

Patterns of help-seeking and treatment did not vary significantly across the groups, with the majority of participants seeking help from a mental health professional or being prescribed medication during their most severe depressive episode (Table 2). Rates of hospital admission during the most severe depressive episode were significantly higher among those with bipolar disorder (46.3\%) compared with major depressive disorder (26.7\%). Rates of any lifetime suicide attempt were comparable among those with bipolar disorder $(32.5 \%)$ and major depressive disorders $(28.3 \%)$. There was no significant difference across the diagnostic 
Table 1 Sociodemographic and clinical characteristics of participants in the major depressive disorder and bipolar type 1 and 2 disorder samples

\begin{tabular}{|c|c|c|c|}
\hline & Unipolar (MDD) $(n=120)$ & Bipolar type $1 / 2(n=246)$ & Test statistic \\
\hline $\begin{array}{l}\text { Gender, }^{a} n(\%) \\
\text { Male } \\
\text { Female }\end{array}$ & $\begin{array}{l}37(30.8) \\
83(69.2)\end{array}$ & $\begin{array}{r}88(36.1) \\
156(63.9)\end{array}$ & $0.98^{\mathrm{b}}$ \\
\hline $\begin{array}{l}\text { Age, years: median (IQR) } \\
\text { At interview } \\
\text { At first mood episode } \\
\text { At first depressive episode } \\
\text { At most severe episode }\end{array}$ & $\begin{array}{l}50(30.5-63) \\
20.5(16-28) \\
20.5(16-28) \\
28(21-41)\end{array}$ & $\begin{array}{l}48(36-57) \\
20(16-29) \\
22(17-31) \\
33(25-42)\end{array}$ & $\begin{array}{r}0.35^{\mathrm{c}} \\
-0.26^{\mathrm{c}} \\
-1.05^{\mathrm{c}} \\
-1.64^{\mathrm{C}}\end{array}$ \\
\hline $\begin{array}{l}\text { Number of lifetime depressive episodes, } n(\%) \\
\text { Five or more episodes }\end{array}$ & $23(26.4)$ & 77 (48.4) & $11.27^{b * *}$ \\
\hline $\begin{array}{l}\text { Duration of most severe depressive episode, } n(\%) \\
\text { Less than } 3 \text { months } \\
3 \text { to } 6 \text { months } \\
\text { More than } 6 \text { months }\end{array}$ & $\begin{array}{l}70(64.8) \\
18(16.7) \\
20(18.5)\end{array}$ & $\begin{array}{r}127(55.2) \\
55(23.9) \\
48(20.9)\end{array}$ & $3.15^{\mathrm{b}}$ \\
\hline $\begin{array}{l}\text { IQR, interquartile range; MDD, major depressive disorder. } \\
\text { a. Total does not equal } 366 \text { because of missing data. } \\
\text { b. Chi-squared test. } \\
\text { C. Mann-Whitney test. } \\
{ }^{* P}<0.05 \text {, }{ }^{* * P}<0.01,{ }^{* * *} P<0.001 \text {. }\end{array}$ & & & \\
\hline
\end{tabular}

\begin{tabular}{|c|c|c|c|}
\hline 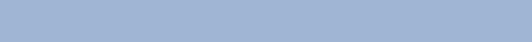 & Unipolar (MDD) $(n=120)$ & Bipolar type $1 / 2(n=246)$ & Test statistic \\
\hline \multicolumn{4}{|l|}{ Treatment, $n(\%)$} \\
\hline Sought help from professional & $94(78.3)$ & $208(84.5)$ & $2.16^{\mathrm{a}}$ \\
\hline Prescribed medication & $84(70.0)$ & $188(76.4)$ & $1.74^{\mathrm{a}}$ \\
\hline Received ECT & $15(12.5)$ & $49(19.9)$ & $3.08^{\mathrm{a}}$ \\
\hline Any treatment & $96(80.0)$ & $211(85.8)$ & $1.99^{\mathrm{a}}$ \\
\hline Hospitalised for depression & $32(26.7)$ & $114(46.3)$ & $13.02^{\mathrm{a} * * *}$ \\
\hline \multicolumn{4}{|l|}{ Suicide history } \\
\hline \multicolumn{4}{|l|}{ Ever attempted suicide, $n(\%)$} \\
\hline Yes & $34(28.3)$ & $80(32.5)$ & $0.66^{\mathrm{a}}$ \\
\hline \multicolumn{4}{|l|}{ Number of attempts, ${ }^{c} n(\%)$} \\
\hline One & $23(67.6)$ & $38(48.7)$ & 3.53 \\
\hline Two & $4(11.8)$ & $17(21.8)$ & \\
\hline Three or more & $7(20.6)$ & $23(29.5)$ & \\
\hline Age at first suicide attempt, years: median (IQR) & $17(16-23)$ & $24.5(17-33)$ & $-1.74^{\mathrm{b}}$ \\
\hline
\end{tabular}

groups in the number of reported attempts or age at first suicide attempt.

\section{Symptom profile during most severe depressive episode}

Table 3 shows the prevalence of each depressive symptom for the major depressive disorder and bipolar disorder groups. In the bivariate analysis the bipolar disorder group was characterised by a significantly greater prevalence (compared with major depressive disorder) of psychomotor retardation, difficulty in thinking or concentrating, early morning wakening, morning worsening, delusions, hallucinations and the presence of three or more concurrent mixed features.

Where a significant bivariate association was found, each symptom was entered into a logistic regression model with diagnosis as the outcome variable. Age- and gender-adjusted odds ratios are reported in Table 4. Compared with the major depressive disorder group, bipolar depression was characterised by significantly higher rates of psychomotor retardation
$(\mathrm{OR}=2.14)$, difficulty thinking $(\mathrm{OR}=2.44)$, early morning wakening $(\mathrm{OR}=1.68)$, morning worsening $(\mathrm{OR}=1.75)$, delusions $(\mathrm{OR}=2.23)$ and hallucinations $(\mathrm{OR}=3.85)$. Mixed features were not significantly associated with bipolar disorder. Each item was then included in a multivariate model to assess the independent association between specific symptoms and diagnostic category after controlling for the effect of other symptoms (Table 4). After adjusting for age, gender and the other items, only psychomotor retardation $(\mathrm{OR}=1.63)$ remained a significant predictor of a bipolar disorder diagnosis compared with major depressive disorder.

\section{Probabilistic approach to the diagnosis of bipolar depression}

To assess the usefulness of the probabilistic approach, we focused on nine clinical features and symptoms that have previously been associated with a bipolar diagnosis, ${ }^{8}$ for which relevant data were available in the version of the DIGS used for this data-set (see Appendix). The number of positive features endorsed by each 
Table 3 Prevalence of depressive symptoms during most severe major depressive episode for participants with major depressive disorder and those with bipolar disorder type 1 or 2

\begin{tabular}{|c|c|c|c|}
\hline & \multicolumn{2}{|c|}{ Prevalence, \% } & \multirow[b]{2}{*}{$\chi^{2}$} \\
\hline & $\begin{array}{c}\text { MDD } \\
(n=120)\end{array}$ & $\begin{array}{c}\text { Bipolar type } 1 / 2 \\
(n=246)\end{array}$ & \\
\hline \multicolumn{4}{|l|}{ Symptoms } \\
\hline Depressed mood & 95.0 & 96.7 & 0.67 \\
\hline Anhedonia & 95.0 & 92.3 & 0.94 \\
\hline Appetite loss & 55.8 & 63.8 & 2.17 \\
\hline Appetite gain & 14.2 & 13.8 & 0.01 \\
\hline Weight loss & 42.5 & 45.9 & 0.38 \\
\hline Weight gain & 10.0 & 15.0 & 1.77 \\
\hline Initial insomnia & 54.2 & 57.7 & 0.41 \\
\hline Middle insomnia & 56.7 & 55.7 & 0.03 \\
\hline Early morning wakening & 43.3 & 54.9 & $4.30^{*}$ \\
\hline Hypersomnia & 35.8 & 45.9 & 3.36 \\
\hline Psychomotor agitation & 46.7 & 50.8 & 0.55 \\
\hline Psychomotor retardation & 38.3 & 55.7 & $9.72^{* * *}$ \\
\hline $\begin{array}{l}\text { Less interest in all pleasurable } \\
\text { things }\end{array}$ & 917 & 927 & 0.12 \\
\hline Fatigue or loss of energy & 84.2 & 89.0 & 1.73 \\
\hline Pathological guilt & 63.3 & 72.8 & 3.39 \\
\hline Worthlessness & 75.8 & 79.7 & 79.7 \\
\hline Difficulty thinking or concentrating & 80.8 & 91.1 & $7.82^{\star *}$ \\
\hline Suicidal ideation & 54.2 & 63.4 & 2.89 \\
\hline Suicide attempt & 22.5 & 29.7 & 2.10 \\
\hline Morning worsening & 38.3 & 52.4 & $6.43^{*}$ \\
\hline Delusions & 12.5 & 23.2 & $5.82^{*}$ \\
\hline Hallucinations & 2.5 & 8.9 & $5.26^{*}$ \\
\hline \multicolumn{4}{|l|}{ Mixed symptoms ${ }^{a}$} \\
\hline Any mixed symptoms & 24.2 & 25.2 & 0.05 \\
\hline Three or more mixed symptoms & 6.7 & 13.8 & $4.06^{*}$ \\
\hline \multicolumn{4}{|c|}{$\begin{array}{l}\text { MDD, major depressive disorder. } \\
\text { a. The Diagnostic Interview for Genetic Studies assessed the following mixed } \\
\text { symptoms during depression episodes: overactive; more talkative/pressured speech; } \\
\text { racing thoughts/speech hard to follow; grandiosity; decreased need for sleep; } \\
\text { distractibilitity risky or indiscreet behaviour. } \\
\star P<0.05, \star * P<0.01, * \star * P<0.001 \text {. }\end{array}$} \\
\hline
\end{tabular}

participant was calculated, and operating characteristics (sensitivity and specificity) were plotted for each possible cut-off (ranging from 0 to 9), with sensitivity decreasing as the number of items included increased. Using a cut-off of three or more items, the probabilistic approach correctly classified $65 \%$ of all participants, with a sensitivity of $73 \%$ and a specificity of $48 \%$. Increasing the cut-off to four or more items saw sensitivity decrease $(48 \%)$ and an increase in specificity (72\%), with $56 \%$ of participants correctly classified. With a more stringent criterion of five or more items, sensitivity dropped to $22 \%$, with specificity reaching $90 \%$. Positive predictive value increased with the number of items, from $74 \%$ with three items to $78 \%$ with four items and $82 \%$ with five items. Participants reporting three or more symptoms were significantly more likely to have been diagnosed with bipolar disorder $(\mathrm{OR}=2.77)$, as were people reporting four or more symptoms $(\mathrm{OR}=2.60)$ and five or more symptoms $(\mathrm{OR}=2.88)$. The area under the curve was 0.63 , indicating that the approach was moderately successful in distinguishing between major depressive disorder and bipolar depression.

\section{Identifying subgroups within major depressive disorder}

The propensity scores (probabilities from the logistic regression) for each of the 120 major depressive disorder cases were entered into a hierarchical cluster analysis, with a two-cluster solution
Table 4 Logistic regression analysis predicting diagnosis

from depressive symptoms

\begin{tabular}{|c|c|c|}
\hline & $\begin{array}{c}\text { Bivariate } \\
\text { analysis }^{\mathrm{a}} \\
\text { OR }(95 \% \mathrm{Cl})\end{array}$ & $\begin{array}{l}\text { Multivariate } \\
\text { analysis }^{\mathrm{b}} \\
\text { OR }(95 \% \mathrm{Cl})\end{array}$ \\
\hline Early morning wakening & $1.68 *(1.07-2.64)$ & $1.37(0.85-2.21)$ \\
\hline Psychomotor retardation & $2.14^{\star *}(1.35-3.38)$ & $1.63^{*}(1.01-2.67)$ \\
\hline Difficult thinking or concentrating & $2.44^{* *}(1.28-4.63)$ & $1.51(0.75-3.02)$ \\
\hline Morning worsening & $1.75^{*}(1.11-2.77)$ & $1.58(0.97-2.59)$ \\
\hline Delusions & $2.23^{*}(1.18-4.22)$ & $1.70(0.87-3.34)$ \\
\hline Hallucinations & $3.85^{*}(1.12-13.21)$ & $2.26(0.63-8.17)$ \\
\hline Mixed episode ${ }^{c}$ & $2.23(0.99-5.03)$ & $2.33(0.99-5.48)$ \\
\hline \multicolumn{3}{|c|}{$\begin{array}{l}\text { a. Each symptom was entered in a separate logistic regression model, adjusted for } \\
\text { age and gender. } \\
\text { b. All seven symptoms were entered into a single logistic regression model, adjusted } \\
\text { for age and gender. } \\
\text { C. Defined by the presence of three or more concurrent hypomanic or manic } \\
\text { symptoms within a major depressive episode. } \\
{ }^{*} P<0.05 \text {, }{ }^{* * P<0.01, ~}{ }^{\star \star * P<0.001} \text {. }\end{array}$} \\
\hline
\end{tabular}

providing the best fit. Cluster 1 consisted of 90 cases, with the remaining 30 cases assigned to cluster 2 . We compared depressive symptoms across the two clusters, with several key differences emerging (Table 5). Cluster 1 was characterised by significantly higher rates of anhedonia, hypersomnia, psychomotor retardation, feelings of worthlessness, difficulty thinking/ concentrating, morning worsening and delusions. In the cluster 1 group, patients were more likely to have reported five or more major depressive episodes and to have been admitted to hospital during their most severe depressive episode. There was no significant difference in other clinical features or symptoms between the two clusters.

\section{Discussion}

A number of robust differences were identified in the phenomenological and longitudinal characteristics of depressive episodes between cases of bipolar disorder and major depressive disorder within these bipolar disorder pedigrees. This is the first

Table 5 Prevalence of symptoms and clinical features showing significant differences between cluster 1 and cluster 2 (major depressive disorder cases only)

\begin{tabular}{|c|c|c|c|}
\hline & $\begin{array}{l}\text { Cluster } 1 \\
(n=90) \\
\%\end{array}$ & $\begin{array}{c}\text { Cluster } 2 \\
(n=30) \\
\%\end{array}$ & $\chi^{2}$ \\
\hline \multicolumn{4}{|l|}{ Symptoms } \\
\hline Anhedonia & 97.8 & 86.7 & $5.9^{*}$ \\
\hline Hypersomnia & 41.1 & 20.0 & $4.4^{*}$ \\
\hline Psychomotor retardation $^{a}$ & 47.8 & 10.0 & $13.6 * * *$ \\
\hline \multicolumn{4}{|l|}{ Less interest in all pleasurable } \\
\hline things & 96.7 & 76.7 & $11.8^{* * *}$ \\
\hline Worthlessness & 81.1 & 60.0 & $5.5^{\star}$ \\
\hline Difficulty thinking or concentrating ${ }^{a}$ & 96.7 & 33.3 & $58.2^{* * *}$ \\
\hline Morning worseninga & 50.0 & 3.3 & $20.7 * * *$ \\
\hline Delusions & 16.7 & 0.0 & $5.7^{*}$ \\
\hline \multicolumn{4}{|l|}{ Clinical features } \\
\hline \multicolumn{4}{|l|}{ Hospitalised } \\
\hline (during most severe MDE) & 31.1 & 13.3 & $3.6^{*}$ \\
\hline Five or more lifetime MDEs & 29.0 & 16.7 & $4.1^{*}$ \\
\hline \multicolumn{4}{|c|}{$\begin{array}{l}\text { MDE, major depressive episode. } \\
\text { a. These symptoms were included in the calculation of the propensity scores using } \\
\text { the full sample, on which the hierarchical cluster analysis was based. } \\
{ }^{*} P<0.05,{ }^{*} P<0.01,{ }^{* *} P<0.001 \text {. }\end{array}$} \\
\hline
\end{tabular}


report comparing depressive features within such families, and supports growing evidence for substantive differences between bipolar and unipolar depression. First, there were a number of differences in the phenomenology of the most severe lifetime depressive episode across these disorders. In cases of bipolar disorder, patients reported a significantly higher prevalence of early morning wakening, morning worsening, psychomotor retardation, difficulty thinking/concentrating, delusions and hallucinations (after controlling for age and gender). Psychomotor retardation was the most robust feature distinguishing between the two groups, supporting previous reports of psychomotor disturbance as a cardinal feature of bipolar depression, ${ }^{19-21}$ as well as historical clinical descriptions. ${ }^{5}$ Psychomotor retardation may have more relevance to bipolar type 1 depression, with Benazzi, for example, finding no difference in rates of retardation between bipolar type 2 disorder and major depressive disorder samples, but rather significantly higher rates of agitation among the group with type 2 bipolar disorder. ${ }^{22}$ In addition, we found a greater prevalence of psychotic features during depressive episodes in the bipolar disorder group, again consistent with numerous prior reports. ${ }^{23}$ The increased frequency of early morning wakening in the bipolar group has previously been reported both in patients with a diagnosis of major depressive disorder who were found to have bipolar disorder on longitudinal follow-up, ${ }^{24}$ and in a number of cross-sectional comparisons with major depressive disorder, ${ }^{23}$ although there have been negative reports. ${ }^{25}$ Overall, the findings are consistent with bipolar disorder being characterised by both melancholic ${ }^{19}$ and psychotic features.

Second, in terms of longitudinal course and treatment, bipolar disorder cases had a greater number of lifetime depressive episodes (a finding which has been widely reported), ${ }^{23,26,27}$ and more hospital admissions, again consistent with previous studies. ${ }^{28,29}$ There was, however, no difference in rates of help-seeking or in usage of antidepressants or electroconvulsive therapy. Despite consistent prior reports of a younger age at onset for patients with bipolar disorder compared with non-related patients with major depressive disorder, ${ }^{30,31}$ no significant difference was found in the age at onset of the first depressive episode in this study. As age at onset of depression has been shown to be highly familial, ${ }^{32,33}$ the lack of a significant difference in this study may be due to the shared family history among cases. In a study comparing probands with bipolar or schizoaffective-bipolar disorder and unwell relatives, there was no difference in the age at onset for the first mood episode between the groups. ${ }^{34}$ Although higher rates of mixed features were observed among cases of bipolar disorder in the initial bivariate comparisons, this difference did not remain significant when logistic regression was used. This finding is perhaps surprising, given reports of the high rates of mixed symptoms in bipolar depression, ${ }^{35}$ although this may further suggest that some clinical differences traditionally seen between major depressive disorder and bipolar disorder were attenuated in the current sample owing to the shared family history.

Third, this study has been the first to empirically test the utility of a proposed probabilistic approach for distinguishing between bipolar and unipolar depression. ${ }^{8}$ Depending on the cut-off employed, the positive predictive value ranged from $74 \%$ to $82 \%$. These values are consistent with or higher than those reported in numerous studies employing the Mood Disorders Questionnaire to differentiate between bipolar and unipolar cases, which have ranged from $36 \%$ to $79 \% .{ }^{36,37}$ In terms of sensitivity and specificity, the optimal cut-off would appear to lie in the range of three to five features; final determination will require validation in independent samples.

\section{Other approaches}

Other methods have been proposed for distinguishing between bipolar disorder and major depressive disorder. Solomon et al developed the Screening Assessment of Depression - Polarity (SAD-P), using three clinical features showing the greatest disparity between patients with the two disorders (number of episodes, family psychiatric history and psychotic features). ${ }^{38}$ This screen achieved a sensitivity of $82 \%$, a specificity of $61 \%$ and a positive predictive value of $36 \%$. In a study of non-psychotic bipolar depression and major depressive disorder involving outpatients recruited to three large multicentre trials (one of bipolar disorder type 1 and two of major depressive disorder), Perlis et al reported that family history, age at onset, number of episodes and a number of items on the Montgomery-Åsberg Depression Rating Scale and Hamilton Rating Scale for Depression differed between these groups. ${ }^{27}$ A stepwise logistic regression was undertaken which correctly classified $87 \%$ of patients, with a sensitivity of $69 \%$ and specificity of $95 \%$. Excluding family history resulted in considerable loss of sensitivity (43\%), highlighting the critical predictive utility of family history in distinguishing between bipolar and major depression. Furthermore, that model has not been tested in independent data-sets.

\section{Identification of subgroups}

The third aim of this study was to test if any differentiating features between bipolar depression and major depressive disorder could be used to identify subgroups within the major depressive disorder sample that might delineate 'genetic' and 'sporadic' populations, i.e. to determine an improved phenotype or case index of underlying bipolarity. Rice has argued that such a 'caseness' index would represent - in the absence of a diagnostic 'gold standard' - a reasonable balance between the strict binary categories of diagnostic systems (such as DSM) and the clinical reality of more equivocal diagnoses. ${ }^{39}$ He suggested that this could be used to weight cases in genetic linkage studies based on the probability that they were truly genetically affected, thereby improving validity of classification and increasing the capacity to identify susceptibility genes. ${ }^{40}$ To our knowledge there has only been one report of a study looking for potential subgroups among major depressive disorder cases in bipolar disorder families. McMahon et al examined age at onset in bipolar disorder pedigrees as an index of genetic liability, finding similarities between those with type 1 and type 2 disorders. ${ }^{9}$ However, there was no similarity in age at onset with the major depressive disorder cases, for which the authors reported a wide variance in age at onset. McMahon et al interpreted this as indicating that the individuals with major depressive disorder represented a heterogeneous population with both genetic and sporadic cases, but it was not possible to statistically identify distinct subgroups. In two case-control studies, Blacker et al compared illness features between unipolar depression in people with a family history of bipolar disorder and that in relatives of those with major depressive illness, ${ }^{14}$ or those in a control group with non-affected families, ${ }^{41}$ but these features showed little discriminatory power in either report. Gershon et al, reporting on depression in relatives of patients with major depressive disorder and bipolar disorder, suggested that greater impairment and multiple lifetime episodes were possible clinical markers of a familial or genetically driven illness, consistent with the higher rates of hospital admission and greater number of lifetime episodes reported here in cluster $1 .{ }^{42}$

We predicted that people with major depression with a postulated 'genetic' illness would be more likely to report features associated with bipolar depression. Using cluster analysis we 
identified two groups, one of which included two-thirds of all major depressive disorder cases and was characterised by higher rates of symptoms found to be associated with bipolar depression in both this study and prior reports. Conversely, the other group demonstrated significantly lower rates of these 'bipolar' depressive features. Clearly this finding suggestive of a genetic subgroup must be regarded cautiously, requiring replication in independent samples before it can be confidently included in phenotypic definitions for linkage or other genetic analyses.

\section{Limitations of the study}

Several limitations should be considered when interpreting the findings of this paper. First, as the study recruited individuals from within bipolar disorder pedigrees, the results may not be generalisable to other populations; the major depressive disorder cases included here are not representative of major depressive disorder in general. Despite this, many findings that have previously been reported in the broader clinical literature on bipolar and unipolar depression were replicated in this genetically defined sample, wherein the shared genetic origins would be expected to diminish the likelihood of demonstrating such differences. Second, the sample size, although consistent with many other comparative studies, was not large enough to enable analysis of the bipolar type 1 and type 2 groups separately. The few studies that have separately examined the two types suggest that there may be important differences between them compared with major depressive disorder. Third, this validation of the probabilistic approach was limited, as not all variables included in the original list operational criteria could be included because of the limitations of the version of the DIGS used in this dataset. Finally, the assessment of depressive features was retrospective, as it was premised on the most severe lifetime episode.

\section{Implications}

In conclusion, a number of clinical features previously associated with bipolar depression were replicated in this sample, which was recruited through bipolar disorder pedigrees. This first empirical validation of the probabilistic approach in differentiating between unipolar and bipolar depression is consistent with dimensional distinctions between the two disorders and appears to offer clinical utility in identifying patients with depression who may warrant further assessment for bipolarity. Finally, cluster analysis of the major depressive disorder sample provided evidence suggestive of subgroups that might represent 'genetic' and 'sporadic' cases - a provocative finding that requires replication in other bipolar disorder pedigree samples.

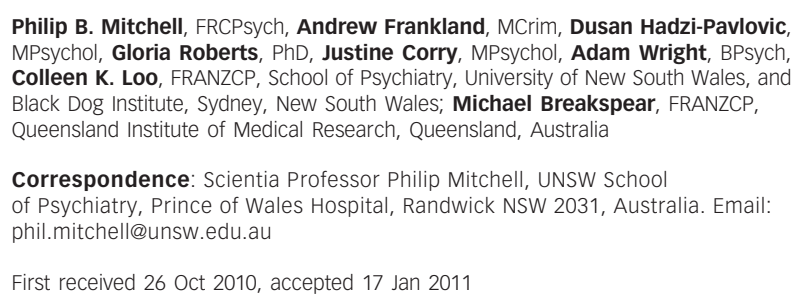

\section{Funding}

This research was funded by Australian National Health and Medical Research Council Program Grant 510135 .

\section{Appendix}

\section{Criteria for a 'probabilistic' approach to the diagnosis of bipolar depression}

A greater likelihood of diagnosis of bipolar type 2 disorder should be considered if five or more of the following features are present:

Symptoms and mental state signs

Hypersomnia a and/or increased daytime napping ${ }^{b}$

Hyperphagia a and/or increased weight ${ }^{\mathrm{a}}$

Other atypical depressive symptoms such as 'leaden paralysis'b

Psychomotor retardation ${ }^{a}$

Psychotic features $^{a}$ and/or pathological guilta

Lability of mood $^{\mathrm{b}} /$ manic symptoms ${ }^{\mathrm{a}}$

\section{Course of illness}

Early onset of first depression ( $<25$ years of age $)^{a}$

Multiple (five or more) prior episodes of depression ${ }^{a}$

\section{Family history}

Family history of bipolar disorder

(Adapted from the criteria published by Mitchell et a $)^{8}$

a. Data were available from the Diagnostic Interview for Genetic Studies (DIGS) to include this symptom in the probabilistic model.

b. No relevant data were available in the DIGS, therefore this symptom was not included in the probabilistic model.

\section{References}

1 Goodwin GM, Anderson I, Arango C, Bowden CL, Henry C, Mitchell PB, et al. ECNP consensus meeting. Bipolar depression. Nice, March 2007. Eur Neuropsychopharmacol 2008; 18: 535-49.

2 Baldessarini RJ, Vieta E, Calabrese JR, Tohen M, Bowden CL. Bipolar depression: overview and commentary. Harv Rev Psychiatry 2010; 18 143-57.

3 Blacker D, Tsuang MT. Unipolar relatives in bipolar pedigrees: are they bipolar? Psychiatr Genet 1993; 3: 5-16.

4 Gershon ES, Hamovit J, Guroff JJ, Dibble E, Leckman JF, Sceery W, et al. A family study of schizoaffective, bipolar I, bipolar II, unipolar, and normal control probands. Arch Gen Psychiatry 1982; 39: 1157-67.

5 Goodwin FK, Jamison K. Manic Depressive IIIness: Bipolar Disorders and Recurrent Depression. Oxford University Press, 2007.

6 Mitchell P, Malhi GS. Bipolar depression: phenomenological overview and clinical characteristics. Bipolar Disord 2004; 6: 530-9.

7 Cuellar AK, Johnson SL, Winters R. Distinctions between bipolar and unipolar depression. Clin Psychol Rev 2005; 25: 307-39.

8 Mitchell PB, Goodwin GM, Johnson G, Hirshfeld RM. Diagnostic guidelines for bipolar depression: a probabilistic approach. Bipolar Disord 2008; 1: 144-52.

9 McMahon F, Stine O, Chase G, Meyers DA, Simpson S, DePaulo J. Influence of clinical subtype, sex, and lineality on age at onset of major affective disorder in a family sample. Am J Psychiatry 1994; 151: 210-5.

10 Judd LL, Schettler PJ, Akiskal HS, Maser JD, Coryell W, Solomon D, et al. Long-term symptomatic status of bipolar I vs. bipolar II disorders. Int J Neuropsychopharmacol 2003; 6: 127-37.

11 Bowden CL. A different depression: clinical distinctions between bipolar and unipolar depression. J Affect Disord 2005; 84: 117-25.

12 Dunner DL. Clinical consequences of under-recognised bipolar spectrum disorder. Bipolar Disord 2003; 5: 456-63.

13 Ghaemi S, Bauer M, Cassidy F, Malhi G, Mitchell P, Phelps J, et al. Diagnostic guidelines for bipolar disorder: a summary of the International Society for Bipolar Disorders Diagnostic Guidelines Task Force Report. Bipolar Disord 2008; 10: 117-28.

14 Blacker D, Lavori PW, Faraone SV, Tsuang MT. Unipolar relatives in bipolar pedigrees: a search for indicators of underlying bipolarity. Am J Med Genet 1993; 48: 192-9. 
15 McAuley E, Blair IP, Liu Z, Fullerton JM, Scimone A, Van Herten M, et al. A genome screen of 35 bipolar affective disorder pedigrees provides significant evidence for a susceptibility locus on chromosome 15q25-26. Mol Psychiatry 2009; 14: 492-500

16 Maxwell M. Family Interview for Genetic Studies (FIGS). National Institute of Mental Health, 1992.

17 Nurnberger J, Blehar M, Kaufmann C, York-Cooker C, Simpson S, Harkavy-Friedman J, et al. Diagnostic interview for genetic studies. Rationale, unique features, and training. NIMH Genetics Initiative. Arch Gen Psychiatry 1994; 51: 849-59.

18 American Psychiatric Association. Diagnostic and Statistical Manual of Mental Disorders (4th edn) (DSM-IV). APA, 1994.

19 Parker G, Roy K, Wilhelm K, Mitchell P, Hadzi-Pavlovic D. The nature of bipolar depression: implications for the definition of melancholia. J Affect Disord 2000; 59: 217-24.

20 Mitchell PB, Wilhelm K, Parker G, Austin MP, Rutgers P, Malhi GS. The clinical features of bipolar depression: a comparison with matched major depressive disorder patients. J Clin Psychiatry 2001; 62: 212-6.

21 Serretti A, Mandelli L, Lattuada E, Cusin C, Smerald E. Clinical and demographic features of mood disorder subtypes. Psychiatry Res 2002; 112: $195-210$.

22 Benazzi F. Symptoms of depression as possible markers of bipolar II disorder. Prog Neuropsychopharmacol Biol Psychiatry 2006; 30: 471-7.

23 Forty L, Smith D, Jones L, Jones I, Caesar S, Cooper C, et al. Clinical differences between bipolar and unipolar depression. Br J Psychiatry 2008; 192: $388-9$.

24 Winokur G, Wesner R. From unipolar depression to bipolar illness: 29 who changed. Acta Neuropsychiatr Scand 1987; 76: 59-63.

25 Duncan WC, Pettigrew KD, Gillin CJ. REM architecture changes in bipolar and unipolar depression. Am J Psychiatry 1979; 136: 1424-7.

26 Schaffer A, Cairney J, Velduizen S, Kurdyak P, Cheung A, Levitt AJ. A population-based analysis of distinguishers of bipolar disorder from major depressive disorder. J Affect Disord 2010; 125: 103-10.

27 Perlis R, Brown E, Baker RW, Nierenberg AA. Clinical features of bipolar depression versus major depressive disorder in large multicenter trials. Am J Psychiatry 2006; 163: 225-31.

28 Goes F, Sadler B, Toolan J, Zamioski R, Mondimore F, Mackinnon D, et al. Psychotic features in bipolar and unipolar depression. Bipolar Disord 2007 9: 907-6.
29 Bottlender R, Jager M, Straub A, Moller H. Suicidality in bipolar compared to unipolar depressed inpatients. Eur Arch Psychiatry Clin Neurosci 2000; 250: 257-61.

30 Abrams R, Taylor MA. A comparison of unipolar and bipolar depressive illness. Am J Psychiatry 1980; 137: 1084-7.

31 Mitchell $\mathrm{P}$, Parker $\mathrm{G}$, Jamieson $\mathrm{K}$, Wilhelm $\mathrm{K}$, Hickie I, Brodaty $\mathrm{H}$, et al. Are there any differences between bipolar and unipolar melancholia? J Affect Disord 1992; 25: 97-105.

32. Schurhoff $F$, Bellivier $F$, Jouvent $R$, Mouren-Simeoni M-C, Bouvard M, Allilaire $J-F$, et al. Early and late onset bipolar disorders: two different forms of manic-depressive illness? J Affect Disord 2000; 58: 215-21.

33 Somanath C, Jain S, Janardhan Reddy Y. A family study of early-onset bipolar I disorder. J Affect Disord 2002; 70: 91-4.

34 Fisfalen ME, Schulze TG, DePaulo J, DeGroot L, Badner JA, McMahon FJ. Familial variation in episode frequency in bipolar affective disorder. Am J Psychiatry 2005; 162: 1266-72.

35 Goldberg JF, Perlis RH, Bowden CL, Thase ME, Miklowitz DJ, Marangell LB, et al. Manic symptoms during depressive episodes in 1380 patients with bipolar disorder: findings from the STEP-BD. Am J Psychiatry 2009; 166: $173-81$

36 Hirschfeld RM, Williams JB, Spitzer RL, Calabrese JR, Flynn L, Keck PE, et al. Development and validation of a screening instrument for bipolar spectrum disorder: the Mood Disorder Questionnaire. Am J Psychiatry 2000; 157: 1873-5.

37 Twiss J, Jones S, Anderson I. Validation of the Mood Disorder Questionnaire for screening for bipolar disorder in a UK sample. J Affect Disord 2008; 110 : $180-4$.

38 Solomon DA, Leon AC, Maser JD, Truman CJ, Coryell W, Endicott J, et al Distinguishing bipolar major depression from unipolar major depression with the Screening Assessment of Depression-Polarity (SAD-P). J Clin Psychiatry 2006; 67: 434-42.

39 Rice JP. Phenotypic definition for genetic studies. Eur Arch Psychiatry Clin Neurosci 1993; 243: 158-63.

40 MacQueen G, Hajek T, Alda M. The phenotype of bipolar disorder: relevance for genetic investigations. Mol Psychiatry 2005; 10: 811-26.

41 Blacker D, Faraone SV, Rosen AE, Guroff JJ, Adams P, Weissman MM, et al. Unipolar relatives in bipolar pedigrees: a search for elusive indicators of underlying bipolarity. Am J Med Genet 1996; 67: 445-54.

42 Gershon ES, Weissman MM, Guroff JJ, Prusoff BA, Leckman JF. Validation of criteria for major depression through controlled family study. J Affect Disord 1986; 11: 125-31. 